\title{
Las redes sociales como generador de valor en las organizaciones
}

\section{Social networks as a generator of value in organizations}

\begin{abstract}
Denises María Ahumada Muñoz. ${ }^{1}$, Andrés Fabricio Cesme Cesme. ${ }^{2}$ \& Washington Edy Santillán Marroquín. ${ }^{3}$
\end{abstract}

\section{Abstract. \\ DOI: https://doi.org/10.33262/visionariodigital.v4i4.1441}

Every day we coexisted with without number of social networks, which, in most of cases facilitate to us to make productive activities and simultaneously, it allows us to communicate yet the planet agilely to us. The social or managed networks, have become a great tool of the organizations, since they help to communicate ideas, to position a mark, to create tendencies or to only inform into fast way, anyway, the communication via social networks of the organizations it establishes an important source of marketing, publicity, administration and human talent. In the present, the positioning and the prestige of the organizations in social networks is very habitual, because great amount of clients and the same competition observes to you with thoroughness and competes creating enterprise more and better contained, which gives the impression that the client perceives better the organization. The generation of big dates via social networks is a remarkable reason that the organizations use to be able to create a base integral of data of clients and thus in the future to be able to fidelizar them through promotions, commercial discounts and other strategies. The innovation of the organizations through the social networks has become a constant that does not stop in the time, the value that the technology by means of apps it offers a the organizations it is fundamental and of great impact, since if she handles of

\footnotetext{
1 Tecnología de Administración de Negocios del Instituto Superior Tecnológico Bernardo O’Higgins., denises.ahumada@gmail.com

2 Tecnología de Administración de Negocios del Instituto Superior Tecnológico Bernardo O’łHiggins., facesme@ohiggins.edu.ec

3 Tecnología de Administración de Negocios del Instituto Superior Tecnológico Bernardo O’Higgins., coordinacion.academica@instituto-ohiggins.com
} 
suitable way and positive the communication and information in these platforms, the organization will be able to blunt successful in these average digitalises.

Key words: Social networks, marketing, publicity, positioning, big date, technology.

\section{Resumen}

Cada día convivimos con un sin número de redes sociales, las cuales, en la mayoría de casos nos facilitan a realizar actividades productivas y a la vez, nos permite comunicarnos ágilmente con todo el planeta. Las redes sociales bien gestionadas, se han convertido en una gran herramienta de las organizaciones, ya que ayudan a comunicar ideas, posicionar una marca, crear tendencias o solamente para informar de manera rápida, de todos modos, la comunicación vía redes sociales de las organizaciones establece una fuente importante de marketing, publicidad, administración y talento humano. En el presente, el posicionamiento y el prestigio de las organizaciones en redes sociales es muy habitual, debido a que gran cantidad de clientes y la misma competencia te observa con detenimiento y compite por crear más y mejor contenido empresarial, el cual da la impresión que el cliente percibe mejor a la organización. La generación de big data vía redes sociales es un motivo notable que utilizan las organizaciones para poder crear una base integral de datos de clientes y así en un futuro poder lograr su fidelidad a través de promociones, descuentos y otras estrategias comerciales. La innovación de las organizaciones a través de las redes sociales se ha convertido en una constante que no se detiene en el tiempo, el valor que la tecnología mediante las apps ofrece a las organizaciones es fundamental y de gran impacto, ya que si se maneja de manera adecuada y positiva la comunicación e información en estas plataformas, la organización podrá despuntar exitosamente en estos medios digitales.

Palabras clave: Redes sociales, marketing, publicidad, posicionamiento, big data, tecnología.

\section{Introducción.}

Para las organizaciones del siglo XXI las redes sociales generan un valor agregado importante, tanto internamente como de manera externa, el poder establecer un segmento de clientes al cual llegar con sus productos o servicios es de vital importancia, esta segmentación ayudará a no perder el norte y no reprocesar y gastar recursos.

La mercadotecnia o marketing digital para la aplicación de estrategias vía redes sociales son un pilar fundamental de comunicación para vender o posicionar un producto o servicio, estas herramientas tecnológicas permiten crear tendencias y establecerse como una organización "top of mind" del mercado, en algunas organizaciones es la única manera de dar a conocer sus productos o servicios, debido a que son plataformas tecnológicas donde se puede llegar aún público diverso, mediante publicidad gratuita, en tiempo record y gran velocidad de respuesta.

Muchas organizaciones optan por crear arte para la difusión de publicidad en programas y plataformas digitales que pagan de manera permanente, pero de igual manera, existen redes 
sociales para la creación de artes, diseños, mejoramiento de fotos, videos e infografías que son utilizadas de manera gratuita.

En el mundo digital existen este momento un sin número de redes sociales, las cuales además de permitir la comunicación, información y publicidad de marcas, productos y servicios, son utilizadas para la atracción del talento humano y de futuros colaboradores para las empresas; en estas redes sociales, la publicación de puestos de trabajo y vacantes son constantes, ya que son plataformas creadas con el fin de publicar y atraer el mejor talento para la organización mediante filtros que ayudaran a seleccionar el candidato ideal y a encontrar las vacantes que se asemejen al perfil del cargo solicitado por los head hunters.

Los clientes externos de una organización son quienes obtienen un relevante beneficio de las redes sociales, el sugerir un cambio o queja mediante la cuenta oficial de la organización es de gran aporte para la alta gerencia, ya que esto ayuda a la mejora continua de procesos y a la conexión más eficaz con el cliente, de esta manera se logrará atender y dar solución inmediata a los diversos requerimientos de los clientes.

El comunicar y utilizar eficientemente las redes sociales para crear y establecer campañas de trabajo social, humano y comercial para la comunidad es algo muy habitual en las organizaciones modernas, estas campañas se las trabaja para generar una buena imagen de la organización frente al cliente externo y para la concientización del público en general, convirtiéndose en un notable vehículo que fortalece la Responsabilidad Social Corporativa de las empresas y comunidad en general.

Dentro de la organización, de igual manera las redes sociales como Whatssap, Instagram y Facebook se han convertido en las plataformas tecnológicas más utilizadas para comunicarse, la rapidez de envío y recepción de mensajes, archivos multimedia, audios y documentos son algunas razones por las cuales estas apps son los sitios de internet favoritos y los más concurridos en el mundo digital.

Luego de mencionar algunas razones por las cuales las redes sociales generan un "valor agregado" a las organizaciones, se establece que el mejoramiento, desarrollo y la capacitación del personal para el manejo de redes sociales es una actividad que hoy en día, mucho más por el COVID-19 se está tomando mucha notoriedad, puesto que las redes sociales disminuyen el tiempo que las personas pasan en los medios de comunicación tradicionales como: la televisión o la clásica radio; por este motivo, no es nuevo o novedoso ver exceso de videos, promociones y descuentos comerciales a menudo en estas plataformas digitales.

Por tal consideración invertir en redes sociales como generador de valor para la organización sigue siendo un recurso inteligente bien pensado y fundamentado. 


\section{Marco Teórico}

La creación de las redes sociales como estrategia comercial, el posicionamiento de la organización y la publicidad, la fase de reclutamiento del talento humano, entre otras opciones, se ha desarrollado significativamente en los últimos años. La comunicación bidireccional entre la organización y cliente es uno de los procesos en los cuales se puede medir la satisfacción del cliente y la percepción integral que éste cliente externo tiene de una determinada compañía.

Existen redes sociales que son más específicas de utilización para ciertas organizaciones, esto depende del sector de mercado en la que se encuentre, así también, de la cultura de la empresa, del país y de los clientes a los cuales se va a llegar, esta elección de cual red social es mejor para cada empresa será determinada por un experto tecnológico en el área y por el departamento de marketing; además, también de los recursos económicos que va a invertir la organización para sus campañas, hay más variables a tomar en cuenta en la elección de una red social para determinada empresa, por ejemplo: el horario de publicitar o comunicar dicha campaña será un aspecto importante a tomar en cuenta, dependerá también de la edad e interés del público, para colocar en una parrilla de horarios focalizados.

Esta es la razón del porqué, un experto en tecnología debería gestionar las fuentes de comunicación vía redes sociales.

La utilización de los medios de comunicación tradicionales se lo está tomando como una segunda opción para comunicar e informar por parte de las organizaciones, se prevé que para el 2030 solo un 13\% de campañas publicitarias serán puestas en televisión, periódico y radio.

Por otro lado, existe una diferencia marcada en el desarrollo, creación y elección de qué medio de comunicación se debe elegir para informar y comunicar, dependiendo del sector de la empresa, sea esta pública o privada, las empresas privadas en la mayoría de casos definen un alto presupuesto en publicidad pagada vía redes sociales, y un porcentaje menor en publicidad en medios tradicionales como revistas, periódicos, televisión y radio; en cambio, las empresas públicas utilizan más los medios tradicionales ya que el estado en algunos países controlan las frecuencias radiales, canales de televisión, revistas y periódicos.

Los avances tecnológicos y el internet dieron vida a la creación las redes sociales, hoy en día por la pandemia, éstas tienen mayor impacto y preponderancia por la velocidad de la comunicación de la información, en cambio, existen empresas que se han consolidado a través de las apps o de plataformas digitales de la información diseñadas y pensadas para el manejo de las preferencias del consumidor.

El caso de Uber y Cabify son casos muy particulares y parecidos, estas empresas nacen en algunos países solo con el dominio de internet y con el lema de dar solución a las necesidades de servicio, atención, seguridad y rapidez que requiere el público de un servicio de taxi. 
Uber y Cabify son ejemplos claros de cómo sacarle el máximo provecho a las redes sociales, en algunos países y también en el Ecuador, estas empresas no disponen de oficinas físicas, instalaciones y peor aún de infraestructura, solo son manejadas remotamente desde una oficina central, la cual constituye el eje operacional de la empresa, de todos modos esta modalidad de negocio y algunas que se asemejan a estas dos ideas serán las que en el futuro predominen y destaquen en la sociedad, porque son de fácil implementación y en algunos países aún no se han creado leyes que regulen este tipo de modalidades de negocio o emprendimiento.

Las ideas de negocio que se implementan a partir de sacarle el máximo provecho al internet, son las mismas que tienen como objetivo retroalimentarse con los usuarios y dar una respuesta y solución adecuada. No debemos dejar de lado el impulso que tomó estos días por la pandemia mundial del Covid-19 "el teletrabajo", es otro esquema de laborar desde la casa utilizando el internet y las plataformas de comunicación digital como Zoom, Google Meet, etc.

El proceso comunicativo organizacional interno es otra opción por la cual los medios digitales son de uso diario en las empresas, el poder conectarse desde cualquier parte del mundo, mediante videoconferencia para estar presentes en decisiones importantes de la organización es una de los motivos por el cual las redes sociales se han convertido en una parte importante de comunicación, la creación de grupos de trabajo en: zoom, whatssap, instagram o facebook son necesarios e indispensables en la mayoría de organizaciones, estos grupos son creados con el propósito de segmentar actividades e implica un ahorro importante de recursos para las empresas.

Internamente, las organizaciones también son atraídas por redes sociales las cuales tienen como fin el de seleccionar y atraer a colaboradores que cubran vacantes de la empresa, estas vacantes serán publicadas en redes sociales como linkedIn, multitrabajos, computrabajos u otros head hunting similares que están orientadas a las empresas, a los negocios y a la búsqueda de empleo, partiendo del perfil de cada candidato para un puesto determinado, que libremente revela su experiencia laboral, formación académica, competencias, logros, etc., la web pone en contacto a millones de empresas y trabajadores con perfiles diversos buscando opciones laborales.

Por ejemplo, mediante filtros linkedin, se ofrece encontrar vacantes de empleos y de puestos de trabajo, los cuales se apeguen al requerimiento de la empresa y al profesional-personal del colaborador de un target medio-alto.

Las organizaciones, al percatarse de la potencialidad que tienen estos nuevos canales de comunicación, han incursionado como entes capaces de relacionarse con sus diferentes públicos utilizando todos y cada uno de los recursos que prestan.

Toda la sinergia de manifestaciones comunicativas es necesaria en la actualidad para lograr una imagen pública importante de cualquier sector de negocio.

Las redes sociales se convierten en un socio estratégico de las organizaciones, se trata de un nuevo y atractivo canal para que cualquier empresa pueda mostrar masivamente sus logros e 
ideas más creativas, conocer a sus públicos de una manera menos formal y sin problemas de estructuras organizativas pesadas.

Este es el caso de las Pymes, las cuales, en su mayoría, mantienen estructuras más pequeñas, flexibles e informales.

El objetivo del presente estudio consiste también en analizar de manera formal si las redes sociales pueden convertirse en instrumentos organizacionales productivos y gestores de identidad y reputación de la marca de una empresa lo que se llama hoy día "branding" es la que define la identidad corporativa de una empresa, que es cuidada de manera celosa por muchas organizaciones en el mundo.

La meta central de este estudio analítico, descriptivo, cuantitativo, es determinar el impacto de las relaciones públicas, humanas y comerciales que se pueden generar a través de las principales redes sociales, considerándolas como parte de los sistemas de información y comunicación empresariales.

Demostrar que las redes sociales son más que una comunidad virtual en donde las personas encuentran, fomentan o interactúan socialmente, y que pueden funcionar dentro de las empresas como una herramienta sustancial en la gestión humana y de negocios de la comunicación empresarial.

Dicha meta, es evaluar el impacto positivo que obtiene una organización por la implementación de las redes sociales como una herramienta comercial, humana y de relaciones públicas.

Esta meta descrita se puede alcanzar, al categorizar las diferentes organizaciones, y sus respectivos públicos y clientes, para después analizar el grado de utilización de las redes sociales mediante la identificación y clasificación de las diferentes aplicaciones y herramientas que las redes sociales tienen en el área de las relaciones públicas y de comunicación interna con el talento humano.

El profesional, con un perfil especializado que maneja las redes sociales en una empresa se lo llama "Comunity Manager" es un profesional de marketing digital responsable de la gestión y desarrollo de la comunidad "online" de una marca o empresa en el mundo digital.

Dentro de sus funciones y responsabilidades de gestión, debe trabajar para aumentar la comunidad de clientes, para detectar a los potenciales clientes y prescriptores. Una vez detectados, establecer con ellos relaciones duraderas y estables, dichas relaciones deberán contribuir a la consecución final de los objetivos de marketing digital de la marca.

El crecimiento de la comunidad en las redes sociales y persuadir positivamente para que el público se identifique con la empresa, es uno de los objetivos del CM o Comunity Manager. 
En este punto, se concentra la atención al cliente, las redes sociales son un escenario perfecto para ello, ya que mediante encuestas creadas en línea, la organización puede tomar correctivos y mejorar continuamente sus procesos, sobre todo aquellos que estén generando malestar en el cliente.

\section{Engagement}

En marketing se lo utiliza para nombrar la implicación emocional que tienen los seguidores con la empresa en las cuentas oficiales de redes sociales de las organizaciones. Mientras el público más comprometido y la interacción con hashtags de la organización sean más frecuente, el trabajo y las estrategias de engagement se las podrá medir de manera más positiva, mediante Kpi's o indicadores de gestión, con herramientas muy potentes como el balanced scorecard y en el caso de métricas de talento humano con el software Compers.

\section{Influencers}

Aunque esta tendencia de publicitar o hacer conocer un producto o servicio mediante la contratación de un personaje famoso o conocido en medios no es nueva, lo que sí se puede aseverar que es nuevo y novedoso es contratar a ejecutivos que cuenten con cierta credibilidad sobre un tema concreto, y por su presencia e influencia en redes sociales puedan llegar a convertirse en un prescriptor interesante para una marca, en esta nueva tendencia de publicitar no quedan de lado los animales influencers, los cuales se los toman como insignia de marcas, campañas y organizaciones para dar a conocer un producto o servicio. Como en todo contexto, se debe realizar estudios de cómo éstos actores conviene más a la empresa, se tomará en cuenta el giro del negocio, por la aceptación de los influencers en los clientes y sobre todo por los seguidores que estos pueden tener en sus redes sociales.

\section{Branding En Redes Sociales (Social Media Branding)}

El social media branding es la gestión específica de la marca en las redes sociales. Es una disciplina dentro del marketing digital que debe estar coordinada con el resto de medios, y que necesita su propia estrategia para conseguir los objetivos de la marca en el consumidor. Cuando se habla de grandes estrategias de branding, se debe mencionar a la empresa APPLE es casi como obligatorio, ya que, esta organización lleva su identidad hasta el máximo, en Instagram han encontrado una mezcla de perfección y conjunción, en sus publicaciones podemos visualizar elegancia, estilo propio, diseño e innovación.

\section{Social Selling}

Es la utilización de las redes sociales por parte de los equipos comerciales de las empresas, para identificar, cualificar y establecer conversaciones con potenciales clientes. Es la manera moderna y actual para desarrollar relaciones significativas con sus prospectos de ventas a fin de 
mantenerle a usted y a su marca en la mente, logrando ser la opción natural de contacto cuando su cliente potencial esté listo para comprar.

\section{Storytelling}

El storytelling es la técnica empleada para contar una historia de una manera distinta, original y siempre desde el punto narrativo. Gracias a esta técnica se puede compartir contenidos que tienen un valor adicional, normalmente ayudan a conectar a nivel psicológico- emocional a las marcas con sus fans. Para que pueda fluir el storytelling necesita de un emisor (marca) y unos oyentes (público). Uno de los casos más reconocidos de Storytelling es el de LEGO, el vídeo dura 17 minutos, y es recomendable verlo cuando tengas tiempo, porque vale la pena, es la historia de la marca contada con un fuerte toque emocional, es imposible no sentirse relacionado e impactado.

\section{¿Para qué utilizamos el Storytelling?}

- Para narrar una historia de forma convincente y efectiva

- Para contar una historia y la a vez entretener a nuestro público

- Para activar la imaginación de nuestro público a través de una historia

- Para narrar una historia que luego el público no pueda evitar compartir

- Para conectarse emocionalmente con nuestra audiencia ¿Qué elementos debemos incluir en el Storytelling?

- El efecto WOW - causa impacto y sorpresa en tu público

- El efecto personal - conecta directamente con su corazón y emociones

- El efecto relevancia - dele algo que de verdad sea relevante, no lo típico de siempre

- El efecto experiencia - vamos a dejarnos de vender mensajes, vamos a vender experiencias prácticas

- El efecto memoria - que nadie se olvide de la historia que has contado

¿Qué Necesitamos para crear Storytelling en redes sociales?

- Objetivos - determinar el fin que quieres conseguir mediante esta técnica

- Una narración - tu mensaje por fases

- Emociones - que tu narración sea capaz de conmover los corazones más fuertes

- Soportes - a través de qué medios vas a trasmitir la historia

- Una moraleja - además de haber vendido por ejemplo tu moto, tienes que trasmitir una moraleja con tu historia.

También en algunos casos es recomendable tener un héroe con el que la audiencia se sienta identificado.

¿Cómo implementamos Storytelling en las redes sociales? 
- Creando una historia a raíz de su marca, por ejemplo, intente mostrar a sus clientes como sería una experiencia con su marca

- Comparta más historias de su marca

- Compartiendo las mejores historias de nuestros clientes de manera conmovedora y convincente.

\section{Resultados.}

Se pudo observar que gran cantidad de casos de influencers son personas que compran seguidores y reacciones, estos bots como se los conoce a seguidores fantasmas son indispensables para el crecimiento de una cuenta en las redes sociales.

De igual manera se encontró que las entidades públicas o gubernamentales son cada vez más las que crean redes sociales y publicitan sus actividades, es así que casi el $45 \%$ de empresas que se encuentran en la región latinoamericana separa anualmente un dinero para promocionarse en redes sociales y así mejorar su imagen, al contrario de las entidades gubernamentales europeas, ya que ahí el presupuesto se lo maneja con auditorias permanentes.

Se calcula que el tiempo promedio que una persona dedica a las redes sociales de la organización son aproximadamente 8 min diarios, este tiempo es compartido en revisar novedades, promociones, comentarios positivos o negativos y a estar atento de la extensión del portafolio de servicios y productos de la organización.

\section{Análisis Cuantitativo.}

Variables a tomar en cuenta en el manejo y creación de redes sociales en las organizaciones

Figura 1. ¿Las redes sociales generan valor en las empresas?

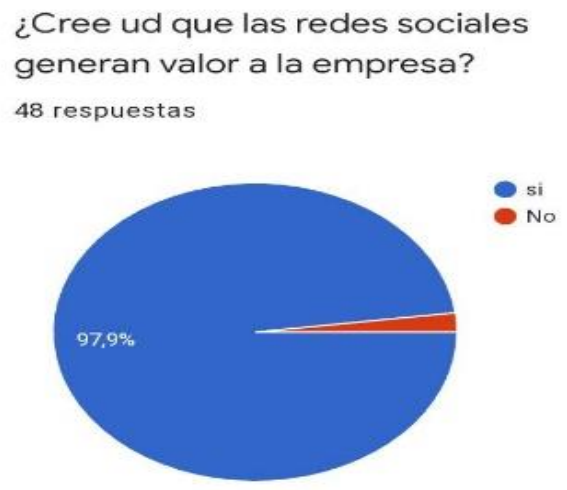

Fuente: Elaboración por los autores 
Figura 2. ¿Qué motiva a escribir en la red social de una empresa?

¿Que le motiva a escribir en las redes sociales de las empresas?

48 respuestas

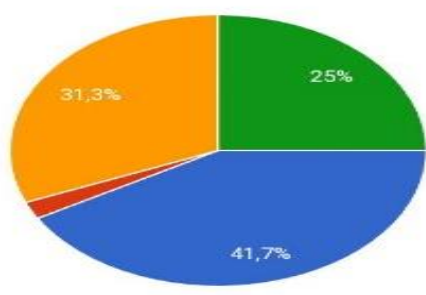

- dejar un comentario

postivio

cojar un negativo

- ayudar a

(a) producto o

- ayudar a fomentar

Fuente: Elaboración por los autores

Figura 3. ¿Ha dejado algún comentario positivo en la red social de una empresa?

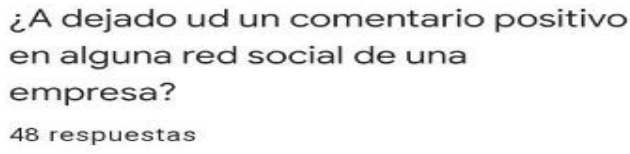

48 respuestas

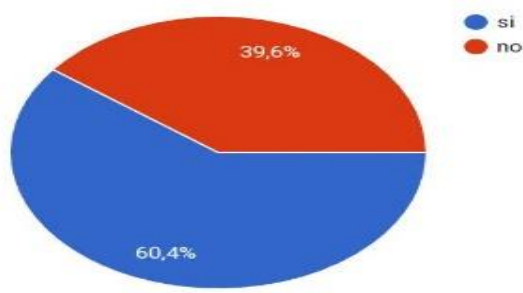

Fuente: Elaboración por los autores

Figura 4. ¿Ha dejado algún comentario negativo en la red social de una empresa?

¿A dejado ud un comentario negativo en alguna red social de una empresa?

48 respuestas

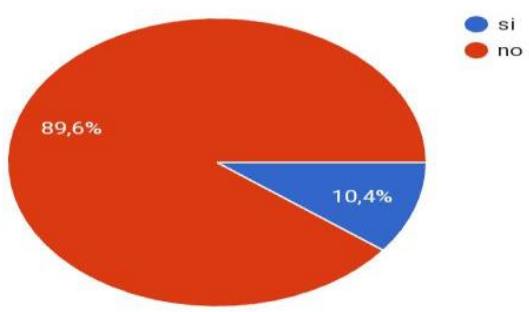

Fuente: Elaboración por los autores 
Figura 5. ¿Por qué sigue las redes sociales de las empresas?

¿Por que le gusta seguir las redes

sociales de las empresas?

48 respuestas

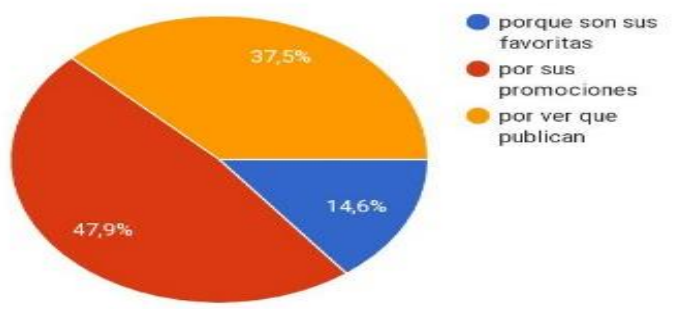

Fuente: Elaboración por los autores

Figura 6. ¿Qué tiempo le dedica a las redes sociales?

¿Que tiempo le dedica a las redes sociales?

48 respuestas

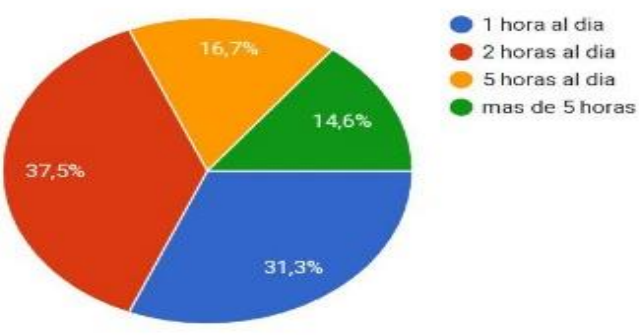

Fuente: Elaboración por los autores

Figura 7. Rango de edades

\section{EDAD}

48 respuestas

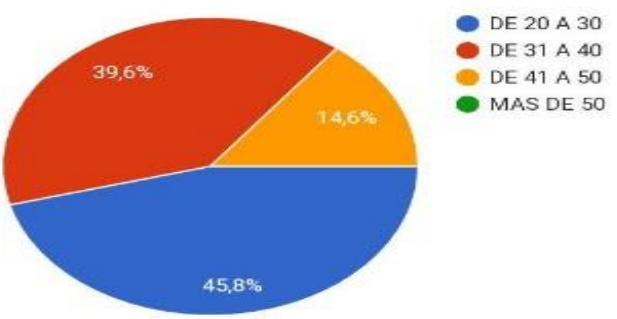

Fuente: Elaboración por los autores 


\section{Discusión.}

Es indiscutible el aprovechamiento de las redes sociales en las organizaciones, ya que a medida que pasa los años, toda red social va mejorando y actualizándose en pro de sus suscriptores, es así que al día de hoy existen plataformas apps y blogs de internet que son más personalizados y mejor pensados para sectores y actividades específicas de negocios.

Las redes sociales así como crean ventajas competitivas a las organizaciones, también crean "dolores de cabeza" en otras, se conoce que los influencers son personas que debido a su magnitud en audiencia congregan muchos seguidores, en ocasiones estos seguidores son falsos, así como sus reacciones para comenta son diversas; es así, que en muchas situaciones han existido empresas estafadas por éstos seguidores fantasmas o seguidores bots, un estudio especializado sobre el tema, titulado "El influencer: herramienta clave en el contexto digital de la publicidad engañosa" realizado por Begoña Gómez Nieto de la Universidad de la Rioja concuerda en que éstos actores son vistos como líderes inalcanzables con cierta credibilidad sobre un tema en concreto, de igual manera se refiere a que los mismos en ciertos temas realizan publicidad engañosa ya que en algunos casos omiten información de lo que publicitan.

Las redes sociales no solo son utilizadas en organizaciones privadas, existen muchas empresas gubernamentales que se atrevieron adentrarse en este mundo digital, estas organizaciones públicas utilizan estos medios para saber qué nivel de aceptación tienen en el público, como ven su gestión, que clase de líderes los considera la gente y también para dar más prestigio a la institución.

En América Latina la cantidad de empresas gubernamentales o públicas que confían en las redes sociales va en aumento, es así como en algunas carteras de estado ya existe un profesional que se dedica al posicionamiento de la entidad, en Europa el manejo de las redes sociales es diferente, ya que debido a sus exigentes y constantes auditorias no tienen tanta libertad para priorizar dinero en redes sociales, hay que recalcar que hay muchas entidades públicas con cuentas oficiales de redes sociales, pero su uso es más informativo y no tanto publicitario.

Es muy importante un manejo adecuado del arte y la manera de saber llegar a través de las palabras en las redes sociales, debido a la expansión de conocimiento y de tecnología hoy los clientes esperan más de las organizaciones en sus cuentas oficiales, es decir, el cliente plantea un mejoramiento constante de lo que la organización publica, este mejoramiento implica rapidez de entendimiento, claridad del tema, honestidad en lo que se quiere llegar a percibir y además una organización más humana, es decir más consiente con sus colaboradores, el planeta y en si con todo lo que le rodea es decir su entorno.

Todo estas variables son de gran importancia al momento de que el usuario de una red social empieza a seguir a la organización, ya que de estas variables depende el compromiso y la lealtad 
del usuario a la cuenta de la organización, si una cuenta organizacional se maneja de mejor manera, esta se convertirá en una fortaleza, el tiempo que el cliente le dedica a visualizar la red social de la organización es muy poco en realidad, es por eso que los mensajes deben ser atractivos, de esta manera se conseguirá que los usuarios se incremente y así poder conseguir posicionar más y de mejor manera a la empresa en el mundo virtual.

\section{Conclusiones.}

- Las redes sociales se han establecido de manera innovadora y muy potente en las organizaciones, cada vez se crean tendencias más efectivas y mejor desarrolladas, esto ayuda a poder obtener el máximo provecho y a lograr los resultados esperados de estas plataformas.

- Se han promovido diversas metodologías de cómo aplicar y de cómo trabajar las redes sociales en las organizaciones, y es claro que, la diversidad de fuentes de trabajo es lo que ha permitido que las redes sociales tengan éxito, no hay una formula predeterminada para el manejo de las redes sociales, esto dependerá de la creatividad y del giro del negocio, incluso si es empresa pública o privada, si es nacional o internacional y de muchos componentes adicionales del sector del mercado en donde se gestiona.

- Lo que se sabe y se conoce con mayor exactitud es que dichas redes sociales son de gran ayuda a las organizaciones, en lograr lealtad en el cliente con la marca o empresa, apoya a la comunicación bidireccional entre cliente y organización y a la necesidad de dar solución inmediata a los requerimientos sociales, humanos o comerciales.

- Al interior de las organizaciones cada día se fundamenta con total positivismo el valor que genera las redes sociales, ya que ayuda a la comunicación interna con todos los niveles de una empresa, mediante estas plataformas la comunicación fluye de mejor manera y cada vez hay menos hermetismos de jerarquía, ya que el agregar un sticker o emoji ayuda eficazmente para quitar la tensión en las comunicaciones y además para poder hacerse entender de mejor manera entre colaboradores y líderes, de todos modos, la comunicación empresarial vía redes sociales se ha transformado de manera positiva y diversa.

- El servicio que brinda una red social a la empresa es gigante, desde una base de datos (big data), identificación de errores, reclutamiento y selección de personal idóneo que cubra un perfil o vacante requerida por el empresa, este último servicio es de gran ayuda para la alta gerencia y los departamentos de talento humano, existen plataformas diversas para encontrar a este talento una más eficiente que otra, pero todas son de gran ayuda, a través de filtros estas plataformas prometen acercarte cada vez al personal adecuado para los objetivos del área y los de la organización. Simplemente te ahorran tiempo y costes económicos.

\section{Referencias Bibliográficas}

Bang. (11 de enero de 2018). Bang! BLOG. Obtenido de 5 Claves para una estrategia de social media Branding: https://bangbranding.com/blog/estrategia-social-media-branding/ 
Bravo, A. (s.f.). Deloitte . Obtenido de La importancia de las redes sociales en las empresas y su gestión: https://www2.deloitte.com/es/es/pages/governance-risk-andcompliance/articles/importancia-redes-sociales-empresas-gestion.html

CitySem. (2020). citySem Dicionario Marketing Digital . Obtenido de Que es Branding: https://citysem.es/que-es/branding/

Dinero. (4 de 11 de 2013). Dinero. Obtenido de ¿Qué marca tiene usted en la cabeza?: https://www.dinero.com/caratula/edicion-impresa/articulo/que-marca-tiene-ustedcabeza/1421

Inbound Cycle. (s.f.). Obtenido de ¿Qué es un influencer? Definición y uso en tu estrategia de marketing: https://www.inboundcycle.com/diccionario-marketing-online/que-es-uninfluencer\#: :text=Un\%20influencer\%20es\%20una\%20persona,plataformas $\% 20 \mathrm{de} \% 20$ v\%C3\%ADdeo\%20como\%20YouTube.

Rios J, Ponce G, Villafuerte D, Espinoza D, \& Tapia K. (2016). Redes sociales como estrategia generadora de valor en las organizaciones. Dialnet, 640-658.

Manuel, J. (01 de 02 de 2018). La Cultura del Marketing. Obtenido de Qué es un Community Manager, funciones y herramientas: https://laculturadelmarketing.com/que-es-uncommunity-manager/

Maram, L. (15 de 05 de 2017). Luismaram Comunicamos Marcas que Inspiran . Obtenido de Qué es social selling; ejemplos y guía para hacerlo: https://www.luismaram.com/quees-social-selling-ejemplos-y-guia-para-hacerlo/

Merca2.0. (26 de mayo de 2015). Mercadotecnia, México, Publicidad. Obtenido de De acuerdo con la agrupación de Taxistas de la Ciudad de México, sus ingresos han disminuido 30 por ciento por Uber y Cabify.: https://www.merca20.com/3-estrategias-de-marketingde-uber/

Milagros, P. A. (2012). Redes sociales, mecanismos generadores de reputación organizacional para la Pyms. Universidad \& Empresa, 131-149.

Miranda, A. M. (2015). Estrategias de marketing con redes sociales en hoteles de cuatro y cinco estrellas en la ciudad de Tijuana, Baja California. Universidad de Quintana Roo, 4.

Nuñez, V. (26 de noviembre de 2013). VN. Obtenido de https://vilmanunez.com/que-es-elstorytelling-y-como-lo-aplicamos-en-: https://vilmanunez.com/que-es-el-storytelling-ycomo-lo-aplicamos-en-redes-sociales/ 
Obeso, P. (19 de diciembre de 2017). rockcontent. Obtenido de Del amor al compromiso: ¿qué es el engagement y cómo medirlo?: https://rockcontent.com/es/blog/medir-elengagement/

Peiro, E. (2020). Marketing Digital. Obtenido de Las 10 tendencias en redes sociales para 2020.

Rodriguez, A, \& Fernandez, A. (2014). Relacion entre el tiempo de uso de las redes sociales en internet y la salud mental en adolecentes colombianos . Acta Colombiana de Psicologia, 131- 140.

Talkwalker. (2020). Obtenido de Tendencias de las Redes Sociales 2021:

https://www.talkwalker.com/es/tendencias-redes-sociales

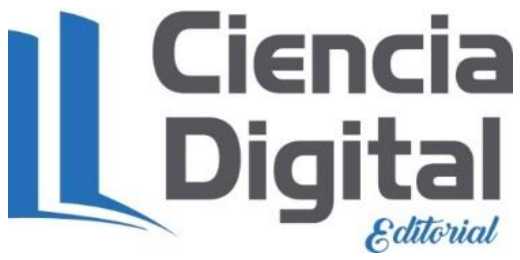


PARA CITAR EL ARTÍCULO INDEXADO.

Ahumada Muñoz, D. M., Cesme Cesme, A. F., \& Santillán Marroquín, W. E. (2020). Las redes sociales como generador de valor en las organizaciones. Visionario Digital, 4(4), 68-81. https://doi.org/10.33262/visionariodigital.v4i4.1441

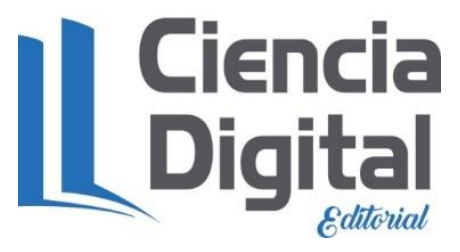

El artículo que se publica es de exclusiva responsabilidad de los autores y no necesariamente reflejan el pensamiento de la Revista Visionario Digital.

El artículo queda en propiedad de la revista y, por tanto, su publicación parcial y/o total en otro medio tiene que ser autorizado por el director de la Revista Visionario Digital.
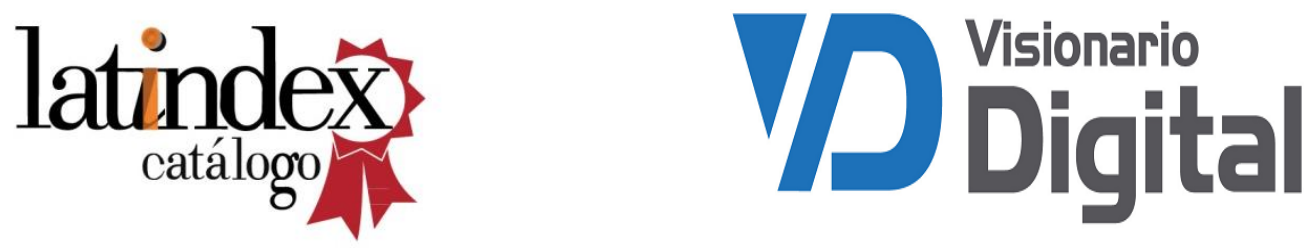